\title{
Safety and Effectiveness of Percutaneous Low-Dose Throm- bin Injection for Femoral Puncture Site Pseudoaneurysms in Neurointervention: Single-Center Experience
}

\author{
Jae Ho Shin, MD, Yunsun Song, MD, Jae Jon Sheen, MD, Dongwhane Lee, MD, Jaewoo Chung, MD, \\ Ga Young Lee, RN, Hyunhee Jeong, RN, Seongsik Han, RT, Joon Ho Choi, RT, \\ Seon Moon Hwang, RT, Deok Hee Lee, MD \\ Department of Radiology, Research Institute of Radiology, Asan Medical Center, University of Ulsan College of Medicine, Seoul, Korea
}

Purpose: We present ultrasound-guided percutaneous low-dose thrombin (200-250 IU) injection for the treatment of iatrogenic femoral pseudoaneurysms. Second, we compared patient and procedure factors between subcutaneous hematoma and pseudoaneurysm groups.

Materials and Methods: From April 2012 to May 2018, 8,425 patients underwent neurointervention. Among these patients, 18 had small subcutaneous hematomas and 6 had pseudoaneurysms. Pseudoaneurysms in the neck and entire sac were visualized, and low-dose thrombins were injected while visualizing a "whirlpool" hyperechoic core in the pseudoaneurysm sac. Subcutaneous hematomas were treated with simple compression. We compared the following parameters between the subcutaneous hematoma group and pseudoaneurysm group: sex, age, body mass index (BMI), type of procedure, heparin usage, sheath size, procedure time, and number of previous neurointervention procedures with the Mann-Whitney $\mathrm{U}$ test.

Results: Most of the pseudoaneurysms were successfully occluded with $200 \mathrm{IU}$ of thrombin $(n=5)$. Only 1 pseudoaneurysm required a slightly higher thrombin concentration $(250 \mathrm{IU}, \mathrm{n=1})$. During the short-term follow-up, no residual sac was observed and no surgical repair was necessary. Pain in the groin region was alleviated. During the 1-month follow-up, no evidence of pseudoaneurysm recurrence nor subcutaneous hematoma was noted. Patient factors (sex, age, and BMI) and procedure factors (heparin usage, sheath size, procedure time, number of previous procedures) were not statistically different between the subcutaneous hematoma and pseudoaneurysm groups.

Conclusion: Ultrasound-guided percutaneous low-dose thrombin injection (200-250 IU) is safe, effective, and less invasive for treating iatrogenic femoral pseudoaneurysm in neurointervention.

Key Words: Thrombin; Arteries; Punctures

\section{Correspondence to: Deok Hee Lee, MD Department of Radiology, Research Institute of Radiology, Asan Medical Center, University of Ulsan College of Medicine, 88 Olympic-ro 43-gil, Songpa-gu, Seoul 05505, Korea Tel: $+82-2-3010-5944$ Fax: +82-2-479-0090 \\ E-mail:dhlee@amc.seoul.kr}

Received: August 24, 2019 Revised: November 22, 2019 Accepted: December 16, 2019

Copyright $\odot 2020$ Korean Society of Interventional Neuroradiology This is an Open Access article distributed under the terms of the Creative Commons Attribution Non-Commercial License (http://creativecommons.org/licenses/by-nc/3.0) which permits unrestricted non-commercial use, distribution, and reproduction in any medium, provided the original work is properly cited. 


\section{INTRODUCTION}

The initial step in neurointervention is the percutaneous puncture of the common femoral artery, which is a frequent access site for diagnostic and therapeutic interventions. ${ }^{1-3}$ latrogenic injury to the femoral artery is common and poses clinical problems. Vascular injury may occur, ranging from mild injuries like subcutaneous hematomas to severe injuries such as pseudoaneurysm, arteriovenous fistula, infection, and retroperitoneal hematoma. ${ }^{1-9}$ Although some of these complications can be managed with simple manual compression and conservative treatment, some complications are life-threatening and require surgery. These also may carry the risk of limb-threatening by thrombosis and ischemic injuries. $^{10-16}$

Recent studies have demonstrated the effectiveness of thrombin injections in treating pseudoaneurysms. ${ }^{17-20}$ In the study by Vlachou et al. ${ }^{17}, 81$ patients were treated with 425 $\mathrm{U} / \mathrm{mL}$ of thrombin with a high success rate of $95 \%$. Another study by Illescas et al. ${ }^{6}$ demonstrated administration of various concentrations of thrombin injections $(50 \mathrm{IU} / \mathrm{mL}$ to 1,000 $\mathrm{IU} / \mathrm{mL}$ ) in 36 patients with a $100 \%$ success rate. Though treatment with variable dosages of thrombin has been reported to be safe and effective, possible thrombotic complications led us to reduce the dosage by $50 \%$ of the currently reported dosages. ${ }^{17-20}$ In this article, we present a single-center experience with ultrasound-guided percutaneous lowdose thrombin (200-250 IU) injection for the treatment of iatrogenic femoral pseudoaneurysm. Second, we compared some factors causing the pseudoaneurysm and subcutaneous hematoma.

\section{MATERIALS AND METHODS}

We conducted a retrospective study of patients who underwent neurointervention. The study was approved by the Institutional Review Board of Asan Medical Center (IRB No. 2019-1437), and we received full consent from patients before the procedures. Between April 2012 to May 2018, 8,425 patients underwent angiographic procedures in our institution (1,741 diagnostic angiographies, 6,684 intervention procedures). We estimated that 350 patients (4.1\%) underwent a surveillance ultrasound examination, partly due to the pain in the groin region and partly owing to the attending physician's discretion. Most of these issues were dismissed as edematous changes without any signs of vascular injuries. Out of these 350 patients, 24 patients had positive findings (pseudoaneurysm, $n=6$; subcutaneous hematomas, $n=18$ ). The diagnosis was made with an ultrasound scanner (HM70A, Samsung Medison, Seongnam, Korea), utilizing a lineal 10.5 $\mathrm{MHz}$ high frequency transducer. We compared the following patient and procedure factors between pseudoaneurysms and subcutaneous hematomas: sex, age, body mass index (BMI), type of procedure, heparin usage, sheath size, procedure time, and number of previous neurointervention procedures. The statistics were calculated with the Mann-Whitney $U$ test.

The thrombin powder (Reyon Pharmaceutical Company, Seoul, Korea) is stored in a designated fridge with an average temperature of $2^{\circ} \mathrm{C}$. A single vial contains 5,000 IU of thrombin extracted from bovine serums. The injection was prepared with a normal saline solution in a $10 \mathrm{~mL}$ syringe. The thrombin powder was diluted such that the $1 \mathrm{~mL}$ of solution contained 1,000 IU of thrombin. Only $0.6 \mathrm{~mL}$ of the solution was extracted, thereby reaching a total of 600 IU. On arrival of the patient, vital signs were measured, and the skin color was closely observed, thereby ruling out the possibility of other co-existing infections or retroperitoneal hematoma.
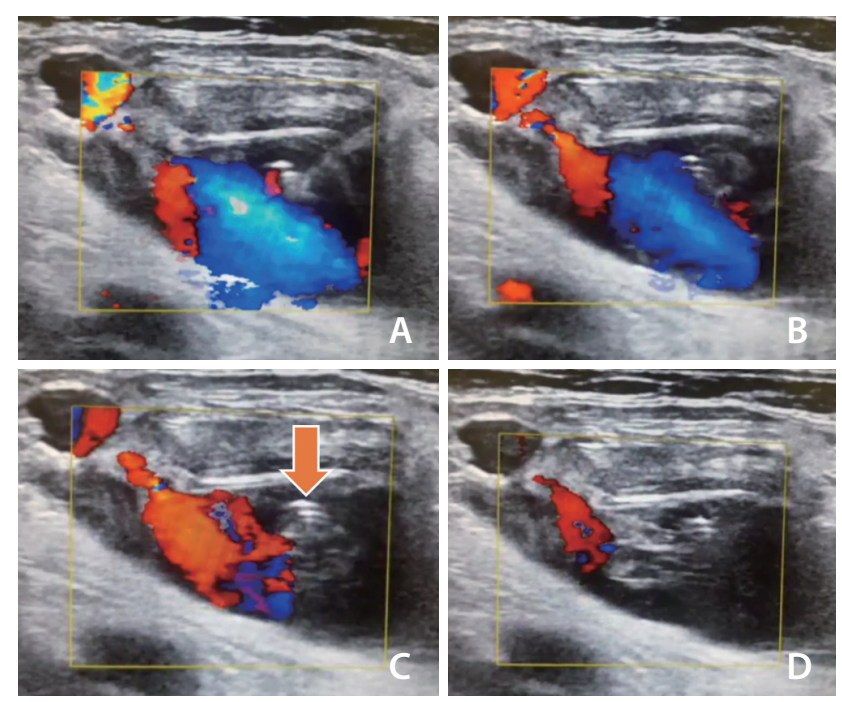

Fig. 1. Ultrasound-guided thrombin injection. (A, B) The most important procedure in thrombin injection is to visualize the entire sac and the location of the pseudoaneurysm neck. Carefully, the low-dose thrombin is injected as far away from the neck as possible. It is vital to keep track of the tip of the needle. (C, D) After a few seconds, swirling patterns of echogenic thrombosis (arrow) are noted. If echogenic cores are not visualized despite the injection, additional injection of $50 \mathrm{IU}$ to $100 \mathrm{IU}$ thrombin can be considered. After completing the procedure, manual compression and absolute bed rest are recommended. 
This was followed by an ultrasound examination.

First, the femoral artery and the puncture site were visualized, ensuring the patency of the punctured femoral artery. Both longitudinal and axial views were then captured, and necessary Doppler wave forms were examined to rule out a possibility of distal or proximal injuries. Second, the pseudoaneurysm was visualized and its neck was carefully traced (Fig. 1). Doppler mode was activated to verify a to-and-fro sign at the sac. Also, fistula tracts were visualized from the sac to the parent artery. At the longitudinal plane, thrombin was carefully injected as far from the neck as possible to avoid inadvertent leakage of the thrombin materials into the main femoral artery (Fig. 2). When the needle tip was confirmed to be within the aneurysmal sac, the thrombin solution (200 IU, $0.2 \mathrm{~mL}$ ) was slowly injected. After a few seconds, swirling echogenic patterns were visualized and propagated within the sac, thereby fully activating thrombogenesis and occlud-
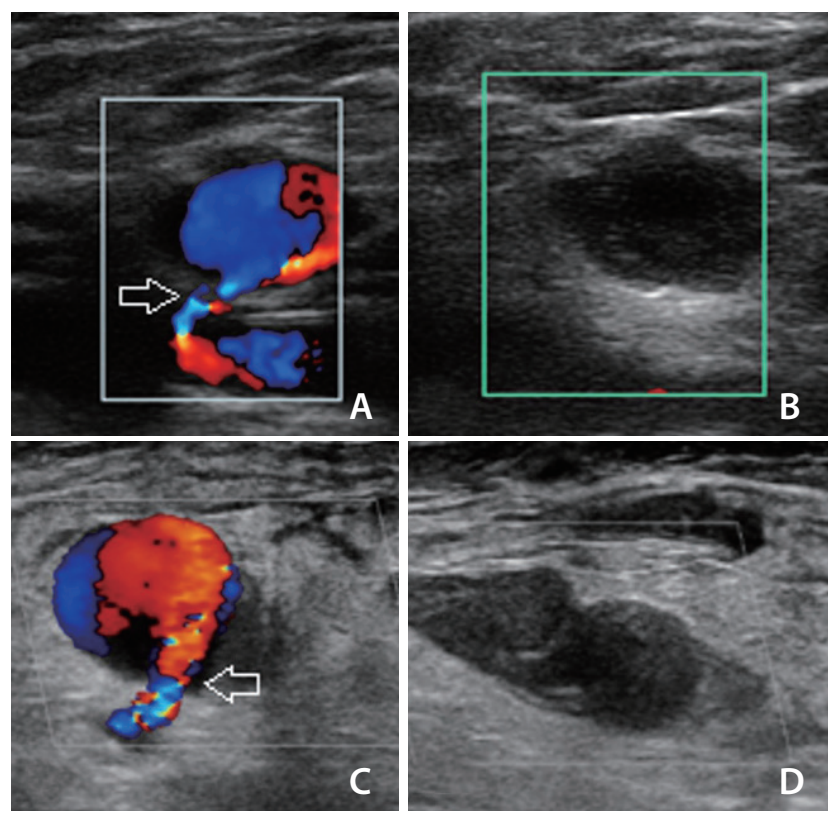

Fig. 2. Thrombogenesis and occlusion within pseudoaneurysms after thrombin injection. (A, B) A 40-year-old male underwent coil embolization and complained of right inguinal pain after 10 days. The ultrasound demonstrated a small pseudoaneurysm in which low-dose thrombin injection successfully occluded the sac without inadvertently leaking the thrombin into the neck (arrow). (C, D) Similarly, a 62-yearold female underwent elective coil embolization, and a pseudoaneurysm was detected after 4 days. The entire sac and neck (arrow) were visualized and thrombin injection was performed, thereby enabling thrombogenesis and occlusion of the pseudoaneurysm. Both the patient's pain and discomfort were alleviated, and follow-up ultrasound before discharge demonstrated no recurrence of the pseudoaneurysm. ing the pseudoaneurysm (Supplementary Video 1). If the swirling patterns were not visualized, we injected additional thrombin (up to $50 \mathrm{IU}$ ) into the sac. We performed follow-up doppler ultrasound within 48 hours to verify the complete resolution of the lesion.

\section{RESULTS}

Accounting for the total patients $(n=8,425)$, only $0.2 \%$ had punctured site complications. Furthermore, only 6 patients had a true pseudoaneurysm, which accounted for $0.07 \%$ of the total procedures during the study duration of 6 years.

The subcutaneous hematoma group $(n=18)$ had the following characteristics. The female to male ratio was 7:11. The average patient age was 61.5 years (range, 47-79). The average BMI was 24.7 (range, 20.9-30.0). Four patients underwent only diagnostic cerebral angiographies $(n=4 / 18)$, whereas 10 patients underwent aneurysm coil embolization $(n=10 / 18)$ and 2 patients underwent stent insertion at the internal carotid artery $(n=2 / 6)$. One patient underwent an emergency drug infusion procedure for a post-clipping vasospasm $(n=1 / 18)$ and 1 patient underwent emergency thrombectomy $(n=1 / 18)$. The median introducer sheath size was $6.0 \mathrm{Fr}$ (range, 4-9). Out of 18 patients, 13 received heparin injection for the procedure. The average procedure time was 109.5 minutes (range, 31.0-181.0). The median number of previous neurointervention procedures was 1.6 (range, $0-4$ ). All of the subcutaneous hematoma patients $(n=18)$ were treated with ultrasonography-guided compression, and follow-up demonstrated no evidence of recurrence or exacerbation of hematomas.

Pseudoaneurysms $(n=6)$ had the following characteristics. The female to male ratio was 5:1. The average patient age was 64.5 years (range, 40-87). The average BMI was 26.8 (range, 20.9-30.0). Two patients underwent only diagnostic cerebral angiographies $(n=2 / 6)$, whereas 4 patients underwent aneurysm coil embolization ( $n=3 / 6)$ and 1 patient underwent stent insertion at the internal carotid artery $(n=1 / 6)$. The median introducer sheath size was 6.0 Fr (range, 4-8). We utilized a $4 \mathrm{~F}$ sheath for diagnostic angiography and a larger bore sheath for the procedure. Out of 6 patients, 4 received heparin for the procedure. The average procedure time was 77.5 minutes (range, 31.0-182.0). The median number of previous neurointervention procedures was 1.8 (range, $0-3)$. The compared parameters were statistically insignifi- 
cant between the subcutaneous hematoma group and the pseudoaneurysm group: proportion of female sex $(61.1 \%$ vs. $83.3 \%, P=0.35$ ), age (61.5 vs. $64.5, P=0.74)$, BMI (24.7 vs. 26.8 , $P=0.28$ ), number of heparin usage (13 vs. $4, P=0.63$ ), sheath size (6 vs. $6, P=0.88$ ), procedure time (109.5 vs. 77.5 minutes, $\mathrm{P}=0.36$ ), number of previous procedures ( 2 vs. $1, \mathrm{P}=0.77$ ).

All of the pseudoaneurysms had their origin from the common femoral artery. Their branches, such as superficial femoral artery and deep femoral artery, were intact. All of the pseudoaneurysm patients had a palpable pulsatile masslike lesion at the right inguinal region, near the puncture site. The average initial diagnosis time from the end of the procedure was 3.5 days (range, 1-10). No patient had a retroperitoneal hematoma nor arteriovenous fistula formations at the puncture site, which were examined with ultrasound and sometimes with a CT scan. The mean dimension of the pseudoaneurysms was $2.0 \times 1.3 \times 1.3 \mathrm{~cm}$ (range, smallest aneurysm: $1.2 \times 1.8 \times 1.1 \mathrm{~cm}$; largest aneurysm: $3.5 \times 1.8 \times 1.5 \mathrm{~cm}$ ). These measurements appear to be small because our measurements only reflected the pure unthrombosed pseudoaneurysm sac.

Most of the pseudoaneurysms were successfully occluded with $200 \mathrm{IU}$ of thrombin $(n=5)$. Only 1 pseudoaneurysm required slightly higher thrombin concentration (250 IU, $n=1$ ). The neck size of all the pseudoaneurysm sacs was less than $1.0 \mathrm{~cm}$, except for 1 patient, who was injected with low dose thrombin (200 IU) at the pseudoaneurysm sac initially but did not form thrombosis within the sac. We then injected an additional $50 \mathrm{IU}$ of thrombin, which subsequently caused thrombosis cascade. After the thrombin injection, all our pseudoaneurysm patients' pain at the right groin region was immediately alleviated as well as the size and extent of the pseudoaneurysms. We also did a short-term follow-up via ultrasound and did not find any recurrence of pseudoaneu- rysms. None of our patients had further surgery or a stent insertion procedure.

\section{DISCUSSION}

Depending on the institution, ultrasound-guided femoral artery cannulations are performed to reduce vascular injury. In our institution, many efforts are made to reduce vascular injuries, such as performing cannulations based on the anatomical demarcations and palpitation at the strongest pulse. We seldom perform ultrasound-guided puncture for patients in whom access to the femoral artery is difficult, such as obese and pediatric patients. After the procedure, we do not utilize a vascular closing device unlike other institutions. Rather, we perform manual compression for a few minutes and apply a compression apparatus that ensures strong compression and sealing of the puncture site.

Even though there are no guidelines, pseudoaneurysm treatments are usually surgical repairs or invasive interventions such as stent insertion. There are less invasive modalities, including ultrasound-guided compression, with success rates ranging between $71 \%$ and $99 \%$. 5 .6 When a complication arises, we always treat patients with manual compression and ultrasound-guided compression. We believe compression should be the first-line treatment for pseudoaneurysms. Unfortunately, ultrasound compression is notoriously difficult and uncomfortable for both the patient and the operator. In our experience, ultrasound-guided compression was time-consuming (average compression time of 30-40 minutes), ineffective (some patients demonstrated recurrence and exacerbating pain), and difficult in treating the pseudoaneurysm (elderly patients were uncooperative due to the pain associated with compression). On the other

Table 1. Various studies on thrombin injection and their outcomes

\begin{tabular}{lccc}
\hline & Thrombin concentration (IU) & Success rate (\%) & Complication rate (\%) \\
\hline Our study & $200(200-250)^{*}$ & 100 & 0 \\
Vlachou et al. $^{17}$ & $425(100-1,500)^{*}$ & 95 & 0 \\
Illescas et al. $^{6}$ & $150(50-1,000)^{*}$ & 100 & 2.2 \\
Reeder et al. $^{18}$ & $192(50-450)^{\dagger}$ & 96 & 0 \\
Paulson et al. $^{14}$ & $306(50-1,600)^{\dagger}$ & 96 & 4.0 \\
La Perna et al. & 100 & 94 & 0 \\
\hline
\end{tabular}

*Data are presented in median concentration and ranges in parentheses.

${ }^{\dagger}$ Data are presented in mean concentration and ranges in parentheses. 
hand, there are numerous reports demonstrating thrombin injection under ultrasound guidance with high success rates, ranging from $93 \%$ to $100 \%$ without serious complications. . $^{5-16}$ Recently, as an alternative to surgical repair and invasive stent insertion, thrombin injection with ultrasound compression has gained much-deserved attention, and there are multiple studies reporting experiences and various concentrations of thrombin injection (Table 1).

There are 2 major contributors to the formation of a pseudoaneurysm: patient-related factors and procedure-related factors. $^{8-11}$ The patient-related factors are BMI, gender, atherosclerotic changes in the punctured artery, age, gender, underlying vascular diseases, and low platelet counts. ${ }^{5-15}$ The procedure-related factors are urgency of the procedure, complexity, duration of the procedure, size of the sheath, and dexterity of the operators and inadequate post-procedure compression. ${ }^{5-15}$ Judging from the previous reports and studies, we assumed that BMl, age, and usage of heparin may increase the occurrence of the pseudoaneurysm as compared to the subcutaneous hematomas. In our institution, however, procedure-related factors and patient-related factors were insignificant when compared to subcutaneous hematomas.

Although thrombin injection has been approved in our institution, there are doubts and unwarranted worries regarding usage of thrombin injection. Different institutions prefer other treatment modalities, such as surgical repair, than thrombin injection, which complicates the situation. Nevertheless, our efforts in minimizing unanticipated complications have shown that low dose thrombin injection is as safe and effective as different modalities and high dose thrombin injection. The technical tip for achieving this is to fully visualize the pseudoaneurysm architecture (sac, neck, and the parent vessel) and slowly inject the thrombin while detecting the thrombosis cascade, or "whirling-pool sign."

Our study has 3 main limitations. First, the sample size was quite small, and we might have underestimated the real number of pseudoaneurysms in our institution. Most of the patients who underwent neurointervention are discharged after a few days, and some of these patients might have experienced a small pseudoaneurysm without being aware of the fact. Often, small pseudoaneurysms occlude spontaneously and pose no clinical problems. Despite the small sample size, we were successful in treating pseudoaneurysms with low dose thrombin injections, and shortterm follow-up demonstrated absolutely no recurrence. The second limitation was the retrospective design of the study and the presence of a non-randomized comparison. The last limitation was that the clinical follow-ups were limited to the short-term, whereas long-term follow-ups are preferred. We hope to perform long-term follow-ups to evaluate any recurrence or complications associated with low-dose thrombin injection.

\section{CONCLUSION}

Ultrasound-guided percutaneous low-dose thrombin injection is a safe, effective, and less invasive method to treat iatrogenic femoral pseudoaneurysms in neurointervention.

\section{SUPPLEMENTARY MATERIALS}

Supplementary materials related to this article can be found online at https://doi.org/10.5469/neuroint.2019.00206.

\section{Fund}

None.

\section{Ethics Statement}

This retrospective study did not receive any forms of funding or grants. The procedures performed in this retrospective study were in accordance with the ethical standards of Asan Medical Center.

\section{Conflicts of Interest}

The authors have no conflicts to disclose.

\section{REFERENCES}

1. Ates M, Sahin S, Konuralp C, Gullu U, Cimen S, Kizilay M, et al. Evaluation of risk factors associated with femoral pseudoaneurysms after cardiac catheterization. J Vasc Surg 2006;43:520-524

2. Dudeck O, Teichgraeber U, Podrabsky P, Lopez Haenninen E, Soerensen $\mathrm{R}$, Ricke J. A randomized trial assessing the value of ultrasound-guided puncture of the femoral artery for interventional investigations. Int J Cardiovasc Imaging 2004;20:363-368

3. Grewe PH, Mügge A, Germing A, Harrer E, Baberg H, Hanefeld $C$, et al. Occlusion of pseudoaneurysms using human or bovine 
thrombin using contrast-enhanced ultrasound guidance. Am J Cardiol 2004;93:1540-1542

4. Gürel K, Gür S, Özkan U, Tekbaş G, Önder H, Oğuzkurt L. US-guided percutaneous thrombin injection of postcatheterization pseudoaneurysms. Diagn Interv Radiol 2012;18:319-325

5. Heis HA, Bani-Hani KE, Elheis MA, Yaghan RJ, Bani-Hani BK. Postcatheterization femoral artery pseudoaneurysms: therapeutic options. A case-controlled study. Int J Surg 2008;6:214-219

6. Illescas MEB, Ruiz AP, Rodríguez MLR, Pardo LC, de la Cruz RQ. Femoral artery pseudoaneurysms. Treatment with thrombin, but ¿always? ECR 2015 Scientific Exhibit. 2015

7. Krueger K, Zaehringer M, Strohe D, Stuetzer H, Boecker J, Lackner K. Postcatheterization Pseudoaneurysm: results of US-guided percutaneous thrombin injection in 240 patients. Radiology 2005;236:1104-1110

8. Kassem HH, Elmahdy MF, Ewis EB, Mahdy SG. Incidence and predictors of post-catheterization femoral artery pseudoaneurysms. Egypt Heart J 2013;65:213-221

9. Knight CG, Healy DA, Thomas RL. Femoral artery pseudoaneurysms: risk factors, prevalence, and treatment options. Ann Vasc Surg 2003;17:503-508

10. La Perna L, Olin JW, Goines D, Childs MB, Ouriel K. Ultrasound-guided thrombin injection for the treatment of postcatheterization pseudoaneurysms. Circulation 2000;102:23912395

11. Tuna Katırcıbaşı M, Güneş H, Çağrı Aykan A, Aksu E, Özgül S. Comparison of ultrasound guidance and conventional method for common femoral artery cannulation: a prospective study of 939 patients. Acta Cardio/ Sin 2018;34:394-398

12. Mishra A, Rao A, Pimpalwar Y. Ultrasound guided percutaneous injection of thrombin: effective technique for treatment of iatrogenic femoral pseudoaneurysms. J Clin Diagn Res 2017;11:TC04-TC06

13. Stone PA, AbuRahma AF, Flaherty SK, Bates MC. Femoral pseudoaneurysms. Vasc Endovascular Surg 2006;40:109-117

14. Paulson EK, Nelson RC, Mayes CE, Sheafor DH, Sketch MH Jr, Kliewer MA. Sonographically guided thrombin injection of iatrogenic femoral pseudoaneurysms: further experience of a single institution. AJR Am J Roentgenol 2001;177:309-316

15. Pinton FA, Spósito AR, Vahle VDA, Soares PR, Zalc S, Perin MA, et al. Ultrasound-guided thrombin injection in the treatment of femoral artery pseudoaneurysm after percutaneous procedures in patients with acute coronary syndrome. Rev Bras Cardiol Invasiva 2013;21:18-22

16. Schneider C, Malisius R, Küchler R, Lampe F, Krause K, Bahlmann $\mathrm{E}$, et al. A prospective study on ultrasound-guided percutaneous thrombin injection for treatment of iatrogenic post-catheterisation femoral pseudoaneurysms. Int J Cardiol 2009;131:356361

17. Vlachou PA, Karkos CD, Bains S, McCarthy MJ, Fishwick G, Bolia A. Percutaneous ultrasound-guided thrombin injection for the treatment of iatrogenic femoral artery pseudoaneurysms. Eur J Radio/ 2011;77:172-174

18. Reeder SB, Widlus DM, Lazinger M. Low-dose thrombin injection to treat iatrogenic femoral artery pseudoaneurysms. AJR Am J Roentgenol 2001;177:595-598

19. Stone PA, Campbell JE, AbuRahma AF. Femoral pseudoaneurysms after percutaneous access. J Vasc Surg 2014;60:1359-1366

20. Yoo T, Starr JE, Go MR, Vaccaro PS, Satiani B, Haurani MJ. UItrasound-guided thrombin injection is a safe and effective treatment for femoral artery pseudoaneurysm in the morbidly obese. Vasc Endovascular Surg 2017;51:368-372 